\title{
A case series of metastatic adrenocortical carcinoma at a tertiary care hospital in UK
}

\author{
F.lqbal, D. Flanagan
}

\section{Plymouth Hospitals NHS Trust}

\section{INTRODUCTION}

Adrenocortical carcinoma is a rare malignancy with an estimated incidence of 0.5-2 new cases per million per year. We present our experience of three patients found with incidental adrenal lesions in a span of approximately 8 months, which were subsequently confirmed to be metastatic adrenocortical carcinoma.

\section{CASE 1}

A 57 year old male was referred to Diabetes Clinic for poor glycaemic control with an $\mathrm{HbA} 1 \mathrm{c}$ of $109 \mathrm{mmol} / \mathrm{mol}$. He reported a six to eight month history of increasing fatigue poor sleep, low mood, leg weakness and a small amount of weight loss as well as thinning and easy bruising of skin. His past medical history was notable for recurrent pancreatitis secondary to chronic alcohol use, including an admission to hospital six months prior for abdominal pain. A CT Abdomen and Pelvis at that admission showed a right adrenal tumour more than $10 \mathrm{~cm}$ in diameter associated with multiple lung nodules and a $2.5 \mathrm{~cm}$ lesion in the liver. On further review, this mass was noted to be present on a CT, done approximately three years ago at which time it measured $6.2 \mathrm{~cm}$ $x 5.9 \mathrm{~cm}$ with a pre contrast density of $-50 \mathrm{HU}$. His workup at that time included one 24 hour urine collection for metanephrines which was normal with unfortunately no further follow up.

At his clinic assessment he was noted to be Cushingoid and the results of his biochemical assessment are as below:

\begin{tabular}{l|l}
24 hour Urinary Free Cortsiol & Sample 1: $2316 \mathrm{nmol} / 24 \mathrm{hr}$ \\
(normal range 0-165 nmol/24 hr) & Sample 2: 1674 nmol/24 hr
\end{tabular}

Overnight Dexamethasone Suppression Cortisol $-615 \mathrm{nmol} / \mathrm{L}$ Test

(normal-cortisol $<50 \mathrm{nmol} / \mathrm{L}$ )

ACTH (normal 0.0-46 ng/L)

His imaging from past (Image $1 a$ \& $1 b$ ) and at presentation (Image $2 a \& 2 b$ ) is shown below:

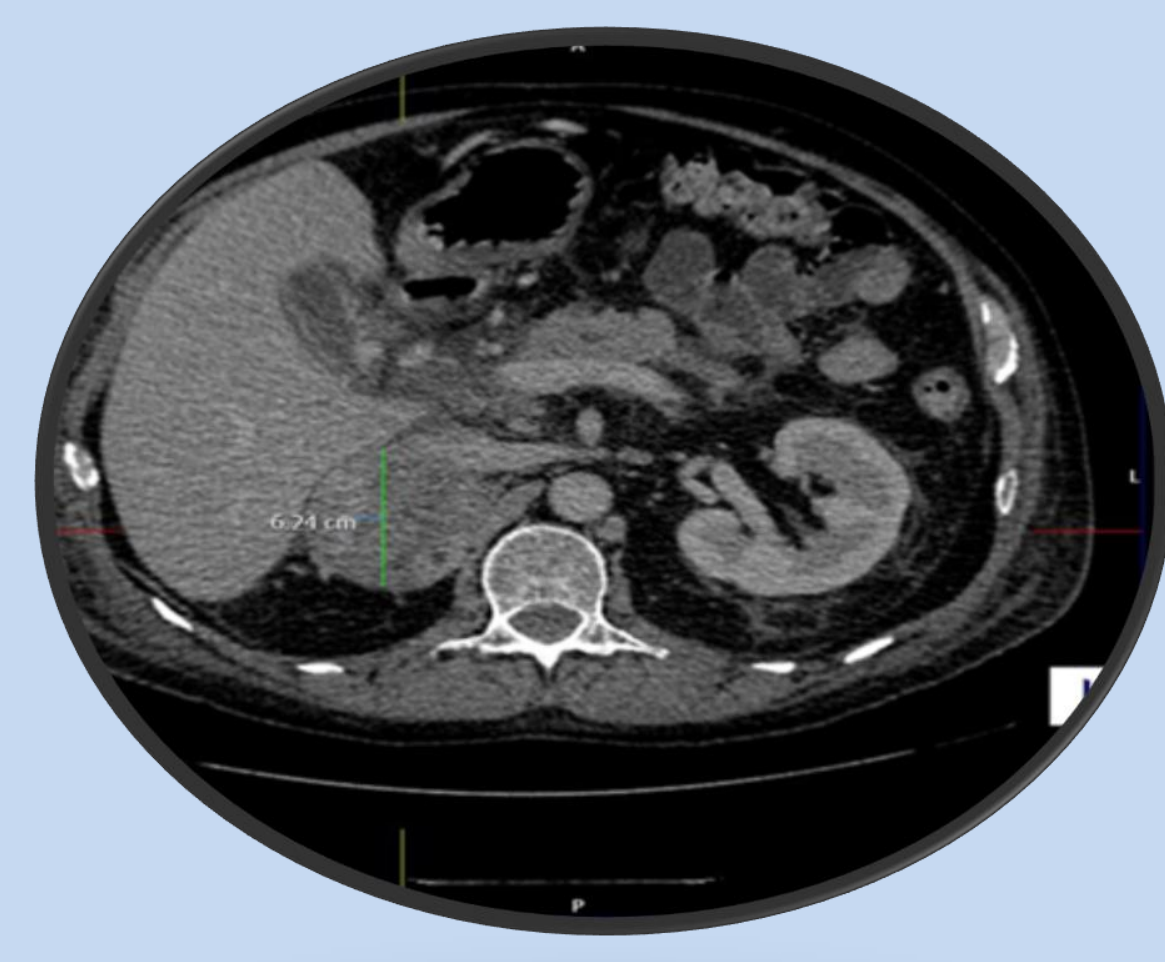

Image 1a

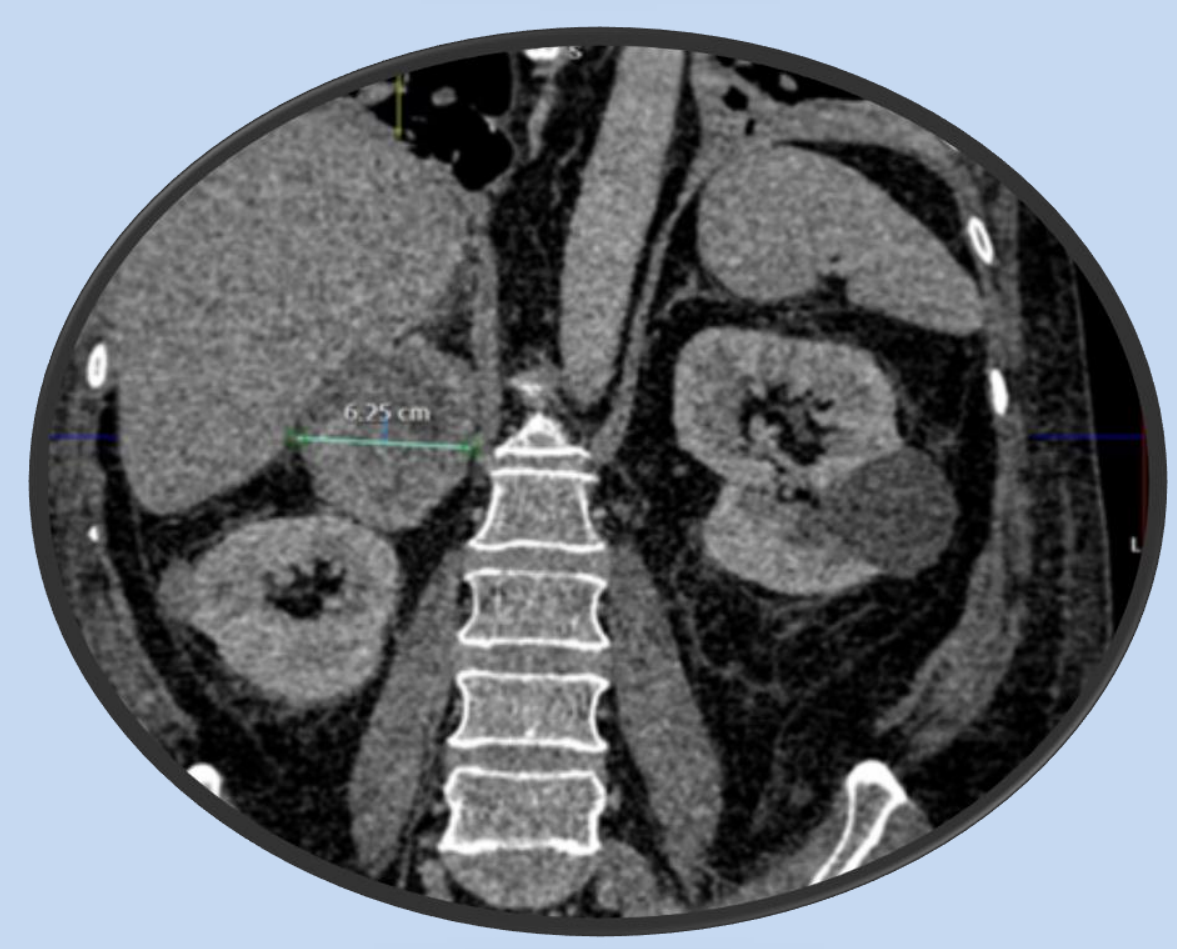

Image $1 b$

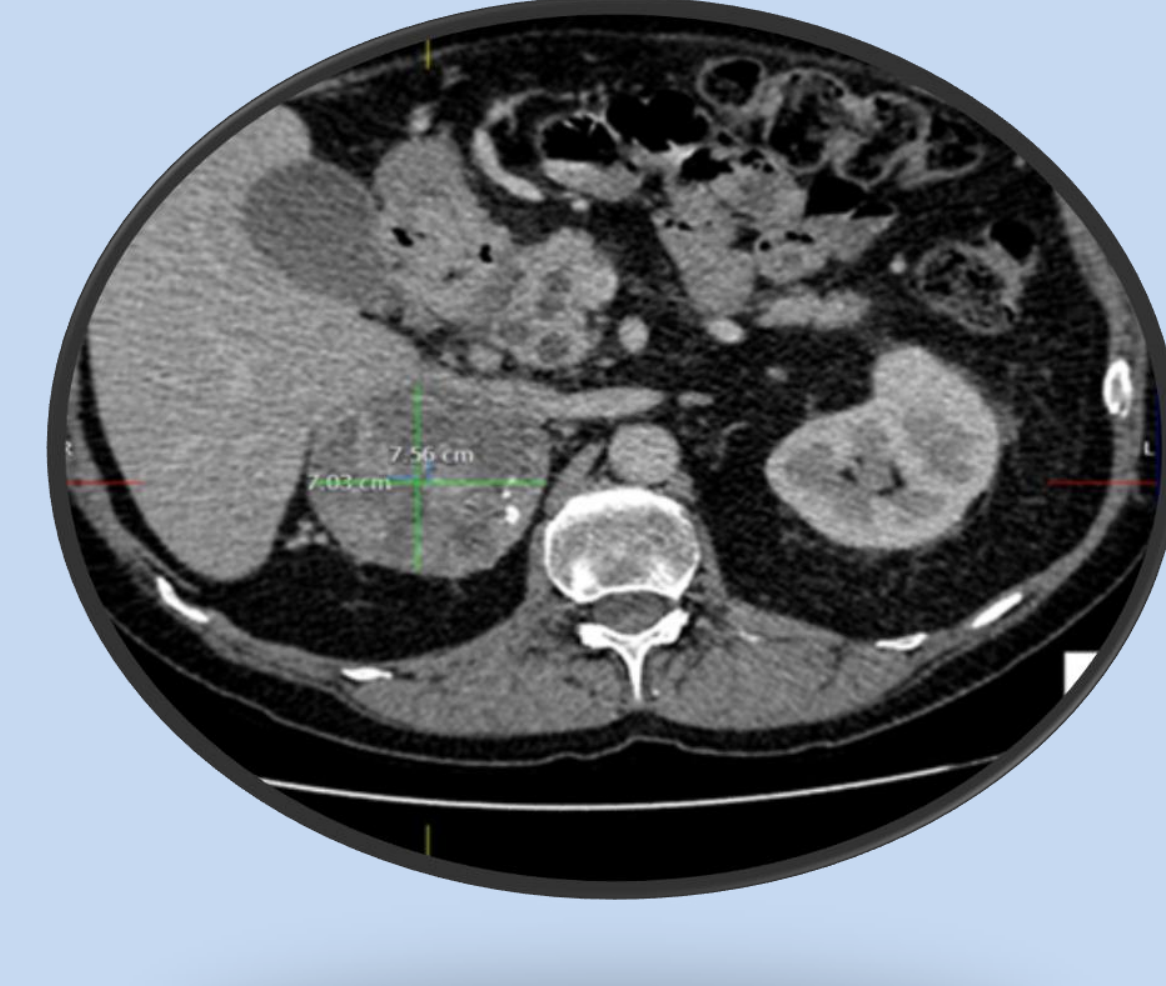

Image 2a

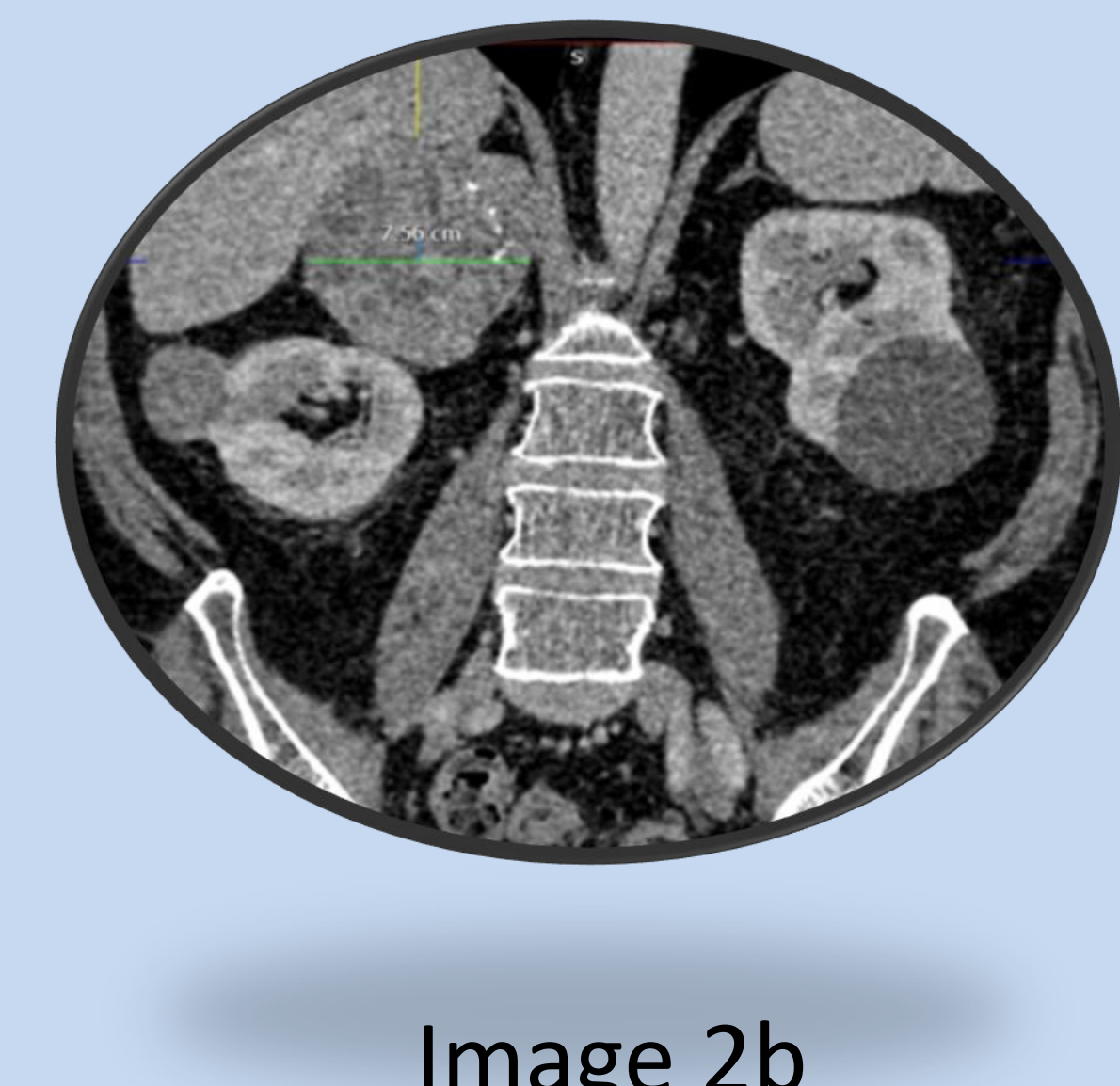

A diagnosis of Cushing's syndrome secondary to metastatic adrenal cancer was made Patient was commenced on metyrapone, and following discussion with Oncology team adjuvant mitotane was added and titrated to a maximum dose of 8 grams/day over a period of four weeks. However, due to development of diarrhoea and severe loss of appetite, the dose was decreased and treatment finally stopped. Follow up scan at 4 months from treatment showed significant progression of the primary tumour as well as increase in pulmonary and hepatic metastases and patient currently under palliative care.

\section{CASE 2}

65 year old male with a history of hypertension was admitted to hospital with high blood sugars, polyuria and polydipsia. He also reported an eighteen month history of severe fatigue, bilateral leg swelling and leg weakness which had left his mobility severely decreased. Initial investigations showed two soft tissue opacities in the right lung, deranged liver function tests and an elevated $\mathrm{HbA} 1 \mathrm{c}$ of $119 \mathrm{mmol} / \mathrm{mol}$. He therefore underwent a CT-Thorax-Abdomen-Pelvis to characterize the lung lesion. This revealed the presence of an $11 \times 10 \mathrm{~cm}$ heterogeneous, partly necrotic and partly solid enhancing mass in the right adrenal gland. A cluster of right lower lobe nodules and a right hilar node were also noted. On clinical examination he was Cushingoid with a plethoric face and neck, protruberant abdomen, bilateral pitting leg oedema and evidence of proximal myopathy. A left foot drop was also noted. An MRI spine was performed and showed upper and lower thoracic endplate compression fractures and a suspicious lesion at $\mathrm{L} 2$. His imaging results and biochemical assessment are given as below:

\section{4 hour Urinary Free Cortsiol \\ (normal range 0-165 nmol/24 hr)}

Overnight Dexamethasone Suppression

Test (normal-cortisol $<50 \mathrm{nmol} / \mathrm{L}$ )

ACTH (normal 0.0-46 ng/L)

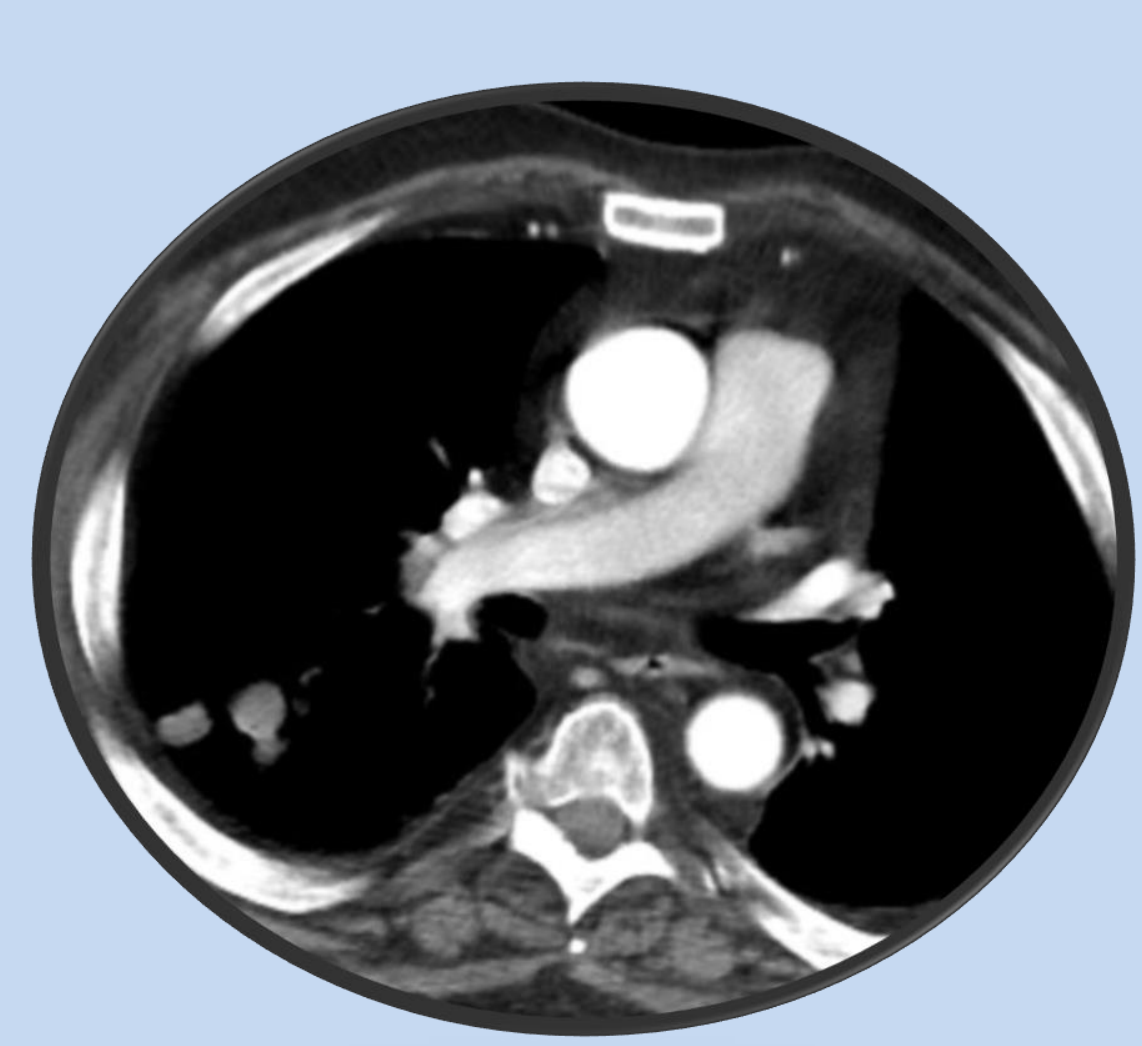

Pulmonary metastasis

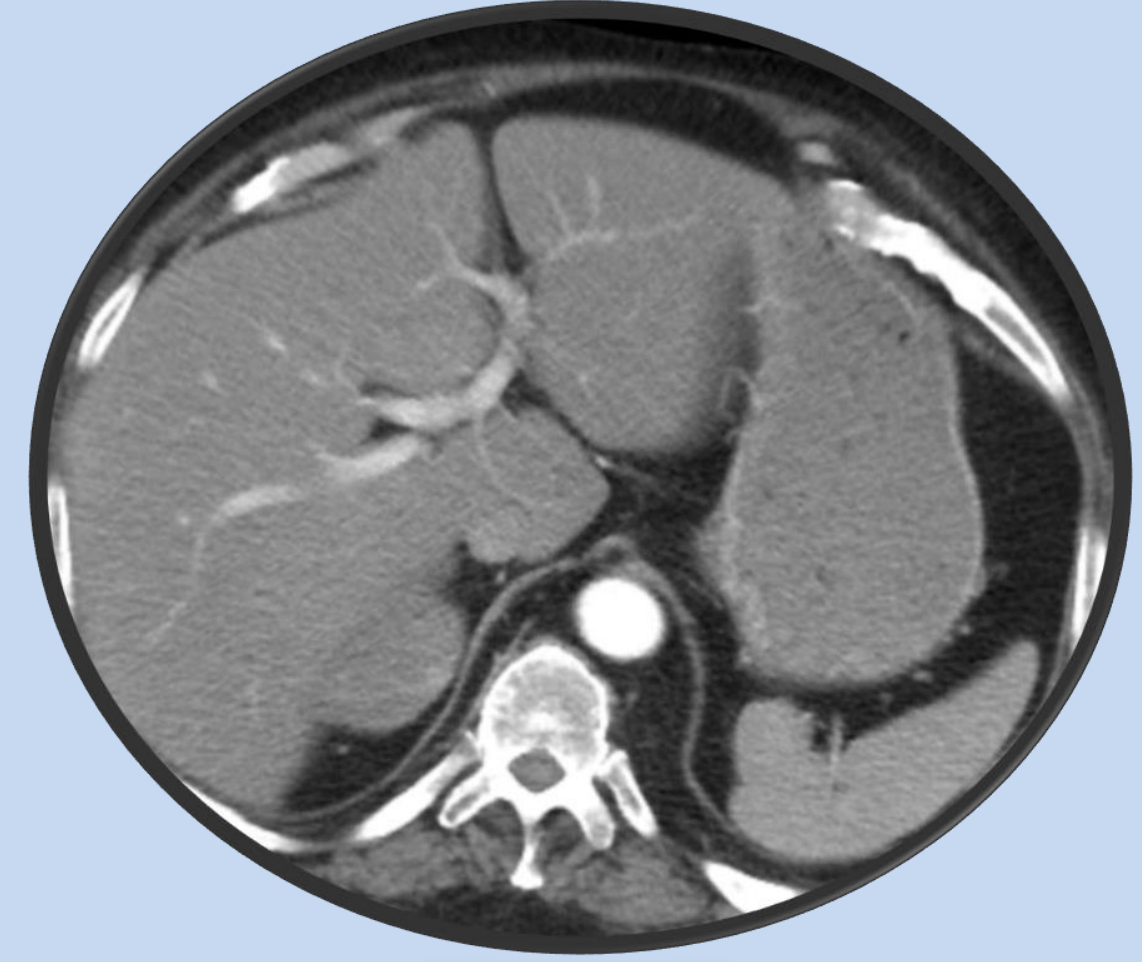

Primary adrenal lesion

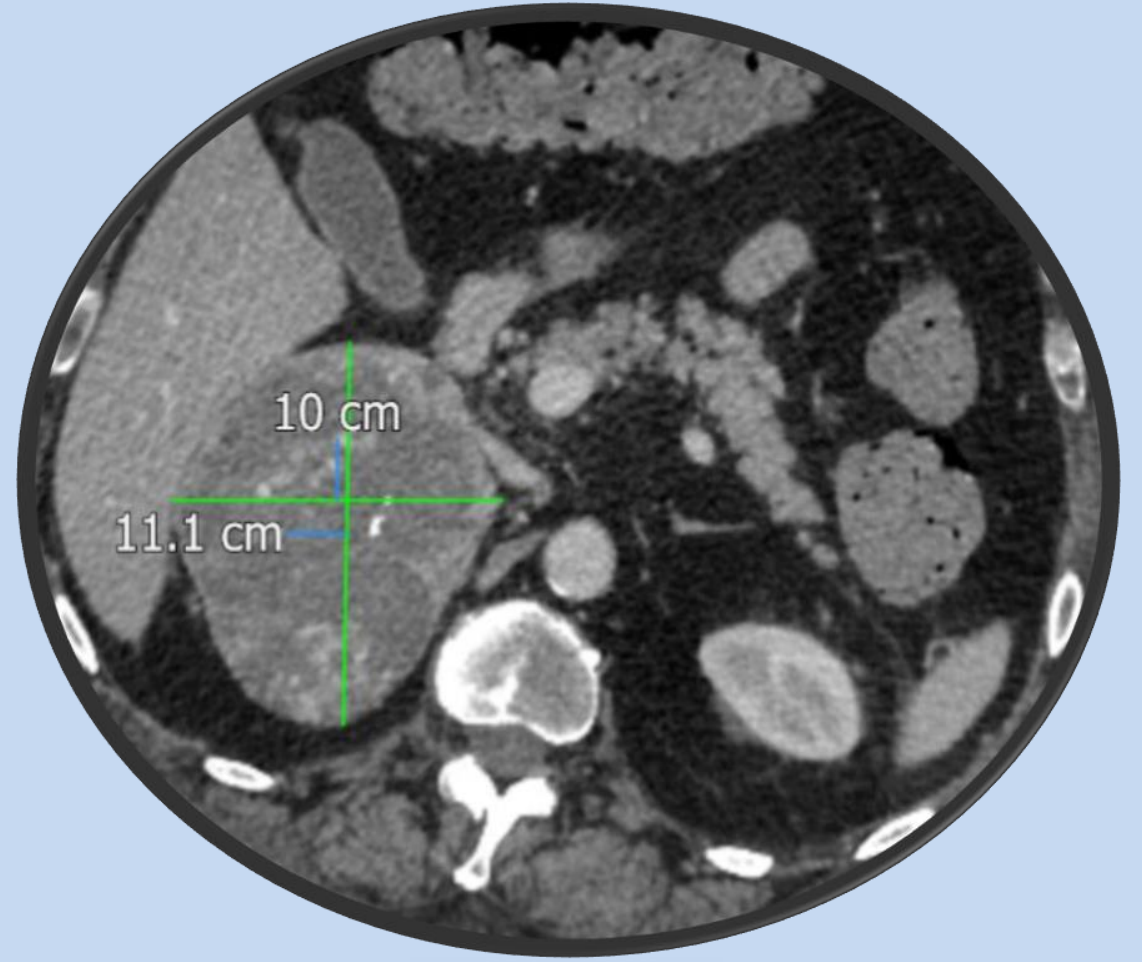

Heterogeneous enhancement of lesion
The patient underwent an Endo Bronchial Ultra Sound with biopsy of pulmonary lesion. Histopathology showed tumour of adrenocortical origin with a MIB1 proliferation index of $25 \%$. PET scan confirmed metabolically active lung and adrenal masses with likely live and lumbar vertebral deposits. Patient started on metyrapone and following two weeks, mitotane commenced and titrated to a maximum dose of 6 grams per day. Unfortunately, during treatment he developed multifocal hospital acquired pneumonia and died following a respiratory arrest.

CASE 3

A 45 year old male presented with sudden onset right sided lower back pain. Imaging showed a $10 \mathrm{~cm}$ heterogeneous, partly cystic/necrotic right adrenal lesion. The spleen was noted to have multiple small ill-defined lesions throughout and an abnormal sclerotic area within right iliac wing was also noted. His past medical history included that of hypertension diagnosed at forty three years of age and recent history of fatigue and night time sweats. His physical examination was unremarkable. PET scan confirmed a large metabolically active right adrenal lesion, while those in the spleen and iliac bone were not metabolically active. Biopsy of iliac wing lesion showed no evidence of neoplasia. His CT (Image 1 ) and SPECT -CT (Image 2) are given below:
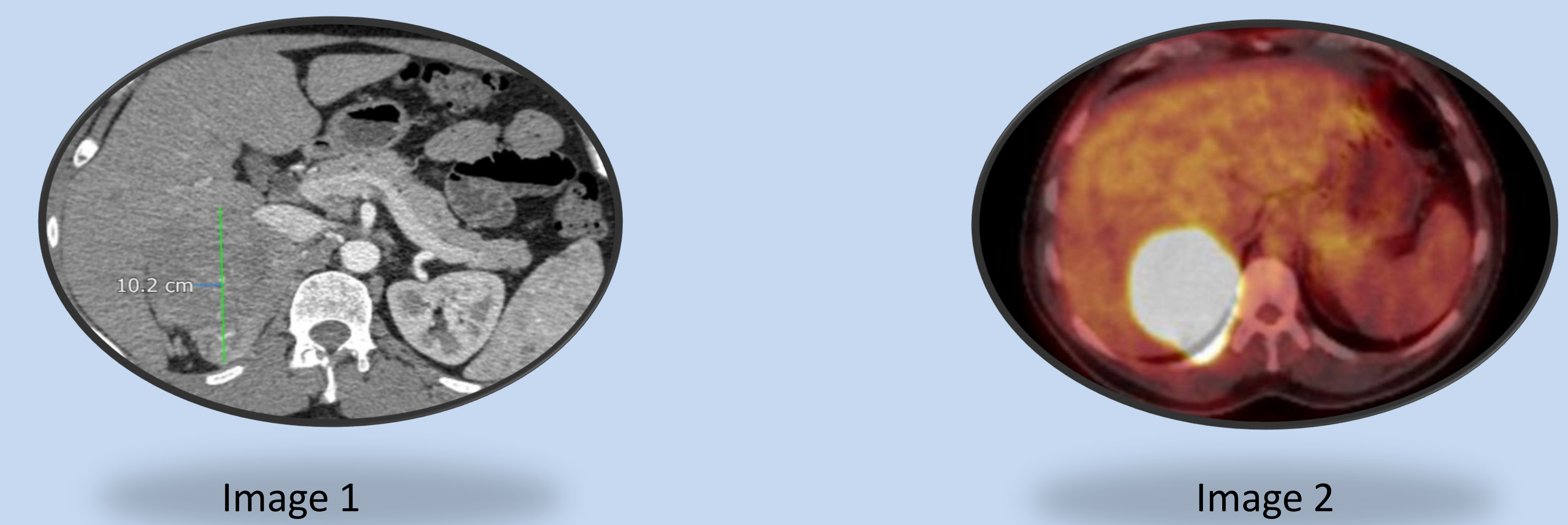

He underwent an urgent adrenalectomy, prior to which two 24-hour urine collections for metanephrines were done and were normal. Histopathology of the resected tumour showed oncocytic adrenocortical carcinoma with a high mitotic rate and atypical mitoses and $\mathrm{Ki}-67$ proliferation index of $40 \%$. Following discussion at MDT, adjunct mitotane therapy was commenced. Unfortunately following eight weeks of treatment with mitotane, he developed fulminant hepatic failure with an ALT of $2119 \mathrm{iu} / \mathrm{L}$, alkaline mitotane, he developed fulminant hepatic failure with an ALT of $2119 \mathrm{iu} / \mathrm{L}$, alkaline
phosphatase $456 \mathrm{iu} / \mathrm{L}$ and bilirubin $87 \mathrm{iu} / \mathrm{L}$. His liver dysfunction was progressive with a continued elevation of his hepatic enzymes and bilirubin above $500 \mathrm{iu} / \mathrm{L}$, precipitating several admissions to hospital. All viral and autoimmune workup for liver failure was negative and liver biopsy showed florid acute hepatitis with pan-acinar necrosis. A diagnosis of drug induced liver injury made and patient commenced on high dose prednisolone treatment.

\section{DISCUSSION}

Adrenocortical carcinoma (ACC) is a rare and highly aggressive malignancy with a bimoda age distribution, having one peak in early childhood and another in the fourth to fifth decade of life. It is more common in females although interestingly all three patients in our series are male. Most patients present either with symptoms of hormone our series are male. Most patients present either with symptoms of hormone mass effects due to tumour growth and extension. Increasingly, however, they may be discovered as incidental lesions during abdominal imaging. On imaging, ACC appears as a heterogeneous mass with irregular margins with or without necrosis, haemorrhage or calcification. The risk for malignancy increases with tumour size, with the index of suspicion increasing for tumours $>4 \mathrm{~cm}$ (sensitivity $97 \%$; specificity $52 \%$ ) and $>6 \mathrm{~cm}$ (sensitivity $91 \%$; specificity $80 \%$ ). Staging of tumour is key to management and the metastasis classification proposed by ESNAT (European Network for the Study of Adrenal Tumours). Age, mitotic count, the proliferation marker Ki67 and glucocorticoid excess are Tumours). Age, mitotic count, the proliferation marker Ki67 and glucocorticoid excess are
additional prognostic markers. For stage 1-3 tumours, surgical resection is treatment of additional prognostic markers. For stage 1-3 tumours, surgical resection is treatment of
choice and it is recommended that adrenal surgery should be performed in selected centres with >10 adrenalectomies for adrenal cancer per year. Adjuvant mitotane or radiotherapy is often used, given the aggressive behaviour and high recurrence rate of ACC. Metastatic disease is associated with a poor prognosis with the 5-year survival being $<15 \%$ Mitotane is the mainstay of treatment in metastatic ACC either alone or in combination with cytotoxic chemotherapy i.e Etoposide Doxorubicin-Cisplatin (EDP). While mitotane is considered one of the most active agents in ACC (response rates ranging from 13 to 35\%) complete responses rarely occur and its use has not shown an increase in overall survival of patients. Though known to be adrenolytic, its full mechanism of anti tumour action is poorly understood. Additionally, there is individual variability in absorption and clearance of this drug with its half life ranging between 18 160 days. It is metabolized and cleared primarily by the liver and induces CYP3A4 activity, however, acute liver failure due to mitotane as seen in one of our patients is rare. Dose limiting side effects include dizziness, vertigo, central nervous system and gastrointestinal side effects.

Given the significant toxicities of mitotane as seen in two of our patients, better tolerated and targeted therapies for metastatic ACC are much needed. 